\title{
Obama catapults patient-empowered Precision Medicine
}

One million or more US citizens will be powering President Barack Obama's Precision Medicine initiative unveiled on January 20 during the State of the Union address. This bold volunteer-driven move to collect and link genotypic, phenotypic and lifestyle data, including crowdsourcing and social media tools, aims to accelerate biomedical discovery with an initial focus on cancer. The patientparticipant cohort at the core of the initiative will enable new approaches to prevention, diagnosis and treatments tailored to individual patients. "It's a new model for doing medical research," says National Institutes of Health's (NIH) director Francis Collins, who spoke in February during a workshop held to discuss the precision medicine approach.

President Obama is seeking an initial \$215 million to launch the program, $\$ 130$ million to build a research cohort of at least a million volunteers and $\$ 70$ million towards the narrower cancer-focused programs within the National Cancer Institute. Another $\$ 10$ million is earmarked for the US Food and Drug Administration, and the remainder to the Office of the National Coordinator for Health Information Technology, to enable the two agencies to work on regulatory policies and bioinformatics capabilities.

This participant-empowered precision medicine initiative is unprecedented, though it builds on recommendations in a 2011 report from the National Research Council (http:// www.nap.edu/catalog/13284/toward-precisionmedicine-building-a-knowledge-network-forbiomedical-research). The plan now is to take two distinct approaches to finding personalized treatments. The first recruits patients with all types of cancer, then selects a targeted drug based on the tumors' genetic abnormalities. The second recruits patients with one type of cancer

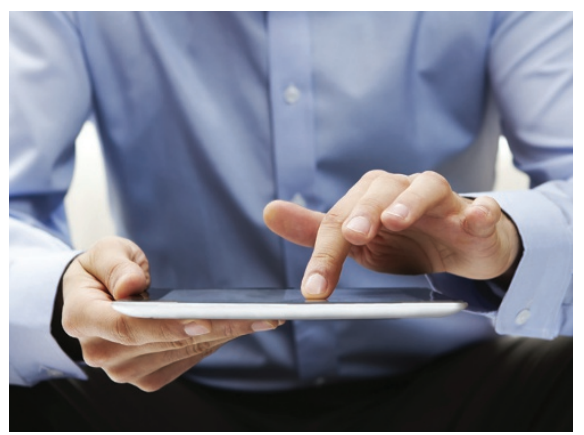

Mobile devices will accelerate medical research.

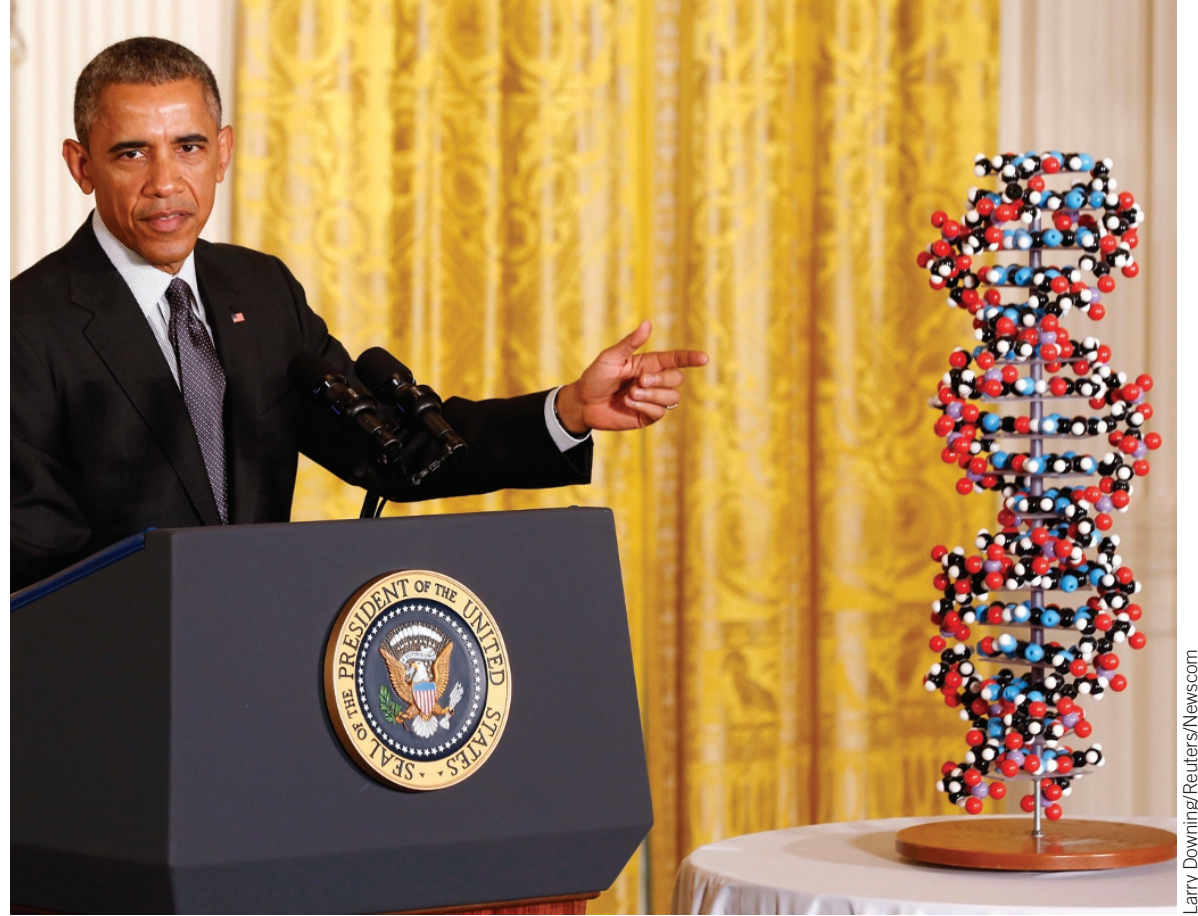

The President's Precision Medicine Initiative aims to deliver genetics-based medical treatments.

and then subdivides them into genomically defined subsets, treating each subset with a different targeted drug.

One looming question is whether to start fresh or to form this huge cohort by building from smaller medical study groups already in place. "It's often more efficient not to be saddled with the old," says workshop participant George Yancopoulos, president and CSO of Regeneron Pharmaceuticals in Tarrytown, New York, who recommends dealing "prospectively" with the "curating and cleaning" challenges that will be needed when analyzing the contents of forthcoming datasets.

$\mathrm{NIH}$ officials envision a grand-scale data-gathering adventure aimed at building the one-millionplus volunteer cohort. But the speed at which genomics datasets are expanding and unresolved issues regarding participants' consent to their electronic medical records remain major challenges. "We don't know how to design this cohort," Collins adds.

One idea is to make volunteering easy through use of social media software resembling Facebook. Another is to share the initiative's design and development features with volunteer participants, including data management responsibilities and funding decisions. If the initiative were to follow a "crowdsourcing mentality to decide priorities," says Anne Wojcicki, CEO of 23andMe in Mountain View, California, with participants owning, and thus controlling, their own data from the outset, decisions about research protocols might move forward faster than with conventional approaches, Wojcicki adds.

In a sense, the initiative launches where genomics leaves off, and some have voiced concerns that it starts another data-driven race, which could be heading in many directions at once. For now, plans for the cohort at the core of the proposed precision medi-

cine initiative go to a subcommittee of the Advisory Committee to the Director at NIH. Perhaps a metaphor best describes the task before that subcommittee. "What we're doing is different from architecture," says workshop participant Douglas Fridsma, president and CEO of the American Medical Informatics Association in Bethesda, Maryland. "It's more like city planning."

Jeffrey L Fox Washington, DC 